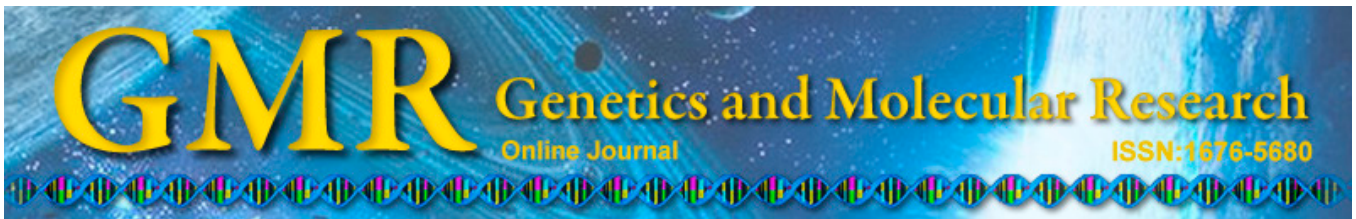

\title{
XRCC3 T241M polymorphism and lung cancer risk in the Han Chinese population: a meta-analysis
}

\author{
J.H. Zhang, ${ }^{1,2}$, Q.L. Wen ${ }^{3}$, C. Yang ${ }^{2}$, A.L. Li ${ }^{2}$, Y. Liu' ${ }^{2}$ and X.S. Li ${ }^{1}$ \\ ${ }^{1}$ Department of Medical Statistics, West China School of Public Health, \\ Sichuan University, Chengdu, China \\ ${ }^{2}$ School of Public Health, Luzhou Medical College, Luzhou, China \\ ${ }^{3}$ Department of Oncology, Affiliated Hospital of Luzhou Medical College, \\ Luzhou, China \\ Corresponding author: X.S. Li \\ E-mail: lixs920@126.com
}

Genet. Mol. Res. 13 (4): 9505-9513 (2014)

Received October 17, 2013

Accepted July 2, 2014

Published November 11, 2014

DOI http://dx.doi.org/10.4238/2014.November.11.15

\begin{abstract}
Numerous studies have evaluated the association between the X-ray repair cross-complementing group 3 (XRCC3) T241M polymorphism and lung cancer risk; however, the actual association is controversial. We examined whether the T241M polymorphism confers a lung cancer risk in China. We searched the PubMed, Google Scholar, and China National Knowledge Infrastructure databases to identify studies that examined the association between the XRCC3 T241M polymorphism and the risk of lung cancer. We estimated the pooled odds ratio with its $95 \%$ confidence interval to assess this association. A total of 3977 patients with lung cancer and 3761 controls from 8 comparative studies were included in this meta-analysis. The meta-analysis results revealed no significant association between the XRCC3 T241M polymorphism and lung cancer risk. In the subgroup analysis, 6 studies with sample sizes over 500 found that the T241M polymorphism had no association with lung cancer. The XRCC3 T241M
\end{abstract}


polymorphism may not be a risk factor for lung cancer. However, larger studies involving a stratified case-control population and biological characterization are needed to validate this finding.

Key words: Lung cancer; Meta-analysis; T241M polymorphism; $\mathrm{XRCC} 3$

\section{INTRODUCTION}

Lung cancer accounts for 13\% (1.6 million) of total cancer cases and 18\% (1.4 million) of cancer-related deaths; it is the most commonly diagnosed cancer and the leading cause of cancer deaths worldwide (Jemal et al., 2011). A previous study reported a 1.63\% increase in lung cancer incidence per year from 1988 to 2005 in China (Chen et al., 2010a); the mortality rate of lung cancer was 30.84 per 100,000 individuals in 2005, representing an increase of $465 \%$ over the past 30 years (Chen et al., 2010b). Previous studies have found several risk factors for lung cancer, including smoking tobacco and being around others' smoke, environmental exposure at home or work (such as radon gas or asbestos), and personal history (such as having radiation therapy or a family history of lung cancer). In addition, genetic factors play an important role in the development of the disease (Wang et al., 2003).

The X-ray repair cross-complementing group 3 gene (XRCC3), a DNA repair gene, codes for a protein that participates in homologous recombination repair of DNA double-strand breaks (DSBs). It is a member of an emerging family of Rad-51-related proteins that may take part in homologous recombination to repair DSBs and maintain integrity of the genome (Brenneman et al., 2000). XRCC3 is localized to human chromosomes 14q32.3. The most frequent polymorphism in $X R C C 3$ is a $\mathrm{C} / \mathrm{T}$ transition that results in an amino acid substitution from Thr to Met at codon 241 (T241M). In addition, variants of the T241M polymorphism may affect the function of the encoded protein to alter DNA repair capacity (Matullo et al., 2001b). Thus, the T241M polymorphism may play a role in the pathogenesis of lung cancer.

In recent years, several studies have been performed to evaluate the relationship between the T241M polymorphism in the XRCC3 gene and lung cancer risk (Liang, 2004, 2005; Zhang et al., 2007; Xia et al., 2008; Qian et al., 2011; Huang et al., 2011; Ke et al., 2012; Guo et al., 2013). However, the results remain controversial, potentially because of small sample sizes, low statistical power, and clinical heterogeneity (Ammar et al., 2012). Therefore, in the present study, we conducted a meta-analysis to examine whether the T241M polymorphism is associated with lung cancer.

\section{MATERIAL AND METHODS}

\section{Selection of studies}

The study retrieval was conducted in PubMed, Google Scholar, and China National Knowledge Infrastructure (CNKI) databases using the search terms "X-ray repair cross-complementing group 3", "XRCC3", "meta-analysis", and "lung cancer" dating up to May 2013. The reference lists of major textbooks, reviews, and included articles were identified through manual searches to find other potentially eligible studies. Studies reported by the same authors, although published in different journals, were checked for possible overlapping partici- 
pant groups. When pertinent data were not included, or data that were presented were unclear, the authors were contacted directly.

\section{Inclusion and exclusion criteria}

The resulting reports were filtered using the following inclusion criteria: 1) casecontrol studies that included lung cancer cases and healthy controls; 2) studies on the association of the XRCC3 T241M polymorphism and susceptibility to lung cancer; 3) studies that included sufficient genotype data for extraction; and 4) healthy controls were in HardyWeinberg equilibrium (HWE). The following studies were excluded: 1) those that were not case-control studies that evaluated the association between the XRCC3 T241M polymorphism and lung cancer risk; 2) case reports, letters, reviews, meta-analyses, and editorial articles; 3 ) reports in which the number of null and wild-type genotypes could not be ascertained; 4) duplicate data were included in the studies; and 5) healthy controls were not in HWE.

\section{Data extraction}

Two investigators (J.H. Zhang and Q.L. Wen) independently extracted the data using a standard protocol and the results were reviewed by a third investigator (C. Yang). Discrepancies were resolved by discussion with our research team. From each article, the following information was extracted: first author, year of publication, region, number of patients and controls, distributions of genotypes and alleles, and evidence of HWE, which are summarized in Table 1.

\section{Statistical analysis}

We calculated the odds ratios (ORs) and corresponding 95\% confidence intervals (CIs) to evaluate the association between the XRCC3 T241M polymorphism and lung cancer risk under a homozygote comparison (TT vs MM), heterozygote comparison (TT vs MT), dominant model (MM+ MT vs TT), and recessive model (TT + MT vs MM) between groups. The effect of heterogeneity was quantified using the $I^{2}$ statistic, which ranges from $0-100 \%$ and represents the proportion of study variability attributable to heterogeneity rather than to chance. $I^{2}$ values of 25,50 , and $75 \%$ were nominally defined as low, moderate, and high estimates, respectively. When $I^{2}>50 \%$, heterogeneity was indicated across studies and the random-effect model was used for meta-analysis; otherwise, the fixed-effect model was used. Before estimating the association between the XRCC $3 \mathrm{~T} 241 \mathrm{M}$ polymorphism and susceptibility to lung cancer, we tested whether the genotype frequencies of controls were in HWE using the $\chi^{2}$ test. Subgroup analysis based on sample size (N subjects $>500$ ) was used to explore and to explain the diversity among the results of different studies. Sensitivity analysis was mainly performed by sequential omission of individual studies or non-HWE studies. We also performed a cumulative meta-analysis to provide a framework for updating the genetic effect of all studies, to measure the extent to which the genetic effect changes as evidence accumulates, and to identify the trend in estimated risk effect (Zintzaras and Lau, 2008). For cumulative meta-analysis, studies were chronologically ordered by publication year, and then the pooled ORs were obtained for each year. Publication bias was investigated using Begg's funnel plot. All statistical analyses were performed using STATA version 10.0 (Stata Corporation, College 
Station, TX, USA). All reported probabilities (P values) were two-sided, with $\mathrm{P}$ values less than 0.05 considered to be statistically significant.

\section{RESULTS}

\section{Study characteristics}

Using our search criteria, 188 individual records were found, and 11 full-text publications were preliminarily identified for further detailed evaluation. Using the exclusion criteria, 3 publications were excluded, including 1 duplicate study and 2 without sufficient data for extraction. As shown in Figure 1, 8 case-control studies examining 3977 lung cancer cases and 3761 controls were included in our meta-analysis (Liang, 2004, 2005; Zhang et al., 2007; Xia et al., 2008; Qian et al., 2011; Huang et al., 2011; Ke et al., 2012; Guo et al., 2013). The main characteristics of eligible studies are summarized in Table 1. In all studies, genotype distributions of controls were in HWE.

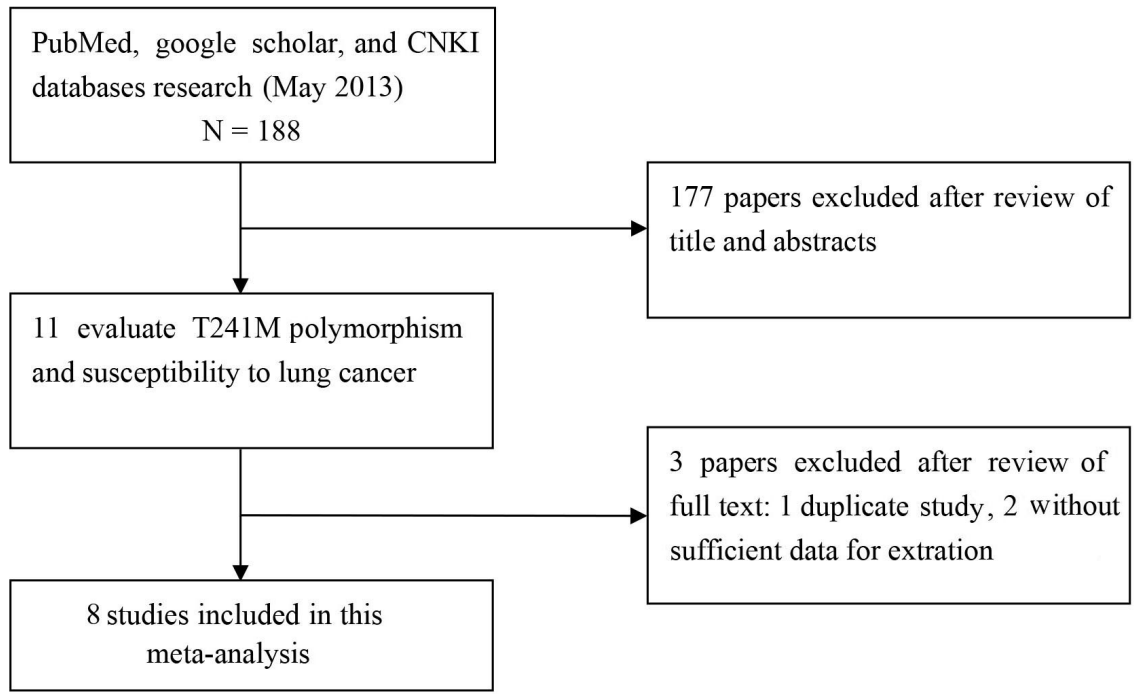

Figure 1. Flow chart showing study selection procedure.

\begin{tabular}{|c|c|c|c|c|c|c|c|c|c|c|}
\hline \multirow[t]{2}{*}{ Study included } & \multirow[t]{2}{*}{ Year } & \multirow[t]{2}{*}{ Area } & \multirow[t]{2}{*}{ Cases/Controls } & \multicolumn{3}{|c|}{ Genotypes for cases } & \multicolumn{3}{|c|}{ Genotypes for controls } & \multirow[t]{2}{*}{ HWE test } \\
\hline & & & & $\mathrm{TT}$ & TM & $\overline{M M}$ & TT & $\mathrm{TM}$ & $\overline{M M}$ & \\
\hline Liang & 2004 & Beijing & $868 / 887$ & 787 & 79 & 2 & 807 & 79 & 1 & 0.51 \\
\hline Liang & 2005 & Jiangshu & $227 / 227$ & 207 & 20 & 0 & 200 & 27 & 0 & 0.34 \\
\hline Zhang et al. & 2007 & Shanghai & $291 / 273$ & 259 & 30 & 2 & 244 & 28 & 1 & 0.84 \\
\hline Xia et al. & 2008 & Zhejiang & $103 / 139$ & 91 & 12 & 0 & 118 & 21 & 0 & 0.34 \\
\hline Qian et al. & 2011 & Tianjin & $581 / 603$ & 521 & 60 & 0 & 533 & 67 & 3 & 0.57 \\
\hline Huang et al. & 2011 & Fujian & $763 / 763$ & 688 & 71 & 4 & 685 & 75 & 3 & 0.54 \\
\hline Ke et al. & 2012 & Jiangshu & $460 / 267$ & 196 & 199 & 64 & 127 & 111 & 29 & 0.52 \\
\hline Guo et al. & 2013 & Shanghai & $684 / 602$ & 589 & 93 & 2 & 549 & 52 & 1 & 0.84 \\
\hline
\end{tabular}




\section{Quantitative synthesis}

The combined results of the T241M polymorphism and lung cancer risk are summarized in Figure 2 and Table 2. The genotype distribution in controls was in HWE (Table 1). The meta-analysis results identified no significant association between the T241M polymorphism and susceptibility to lung cancer in Chinese populations (TT vs MM: OR $=0.74$, $95 \% \mathrm{CI}=0.49-1.14$; $\mathrm{TT} v s \mathrm{MT}: \mathrm{OR}=0.94,95 \% \mathrm{CI}=0.82-1.08$; dominant model: $\mathrm{OR}=$ $1.08,95 \% \mathrm{CI}=0.94-1.24$; recessive model: $\mathrm{OR}=0.79,95 \% \mathrm{CI}=0.52-1.19)$. In the stratified analysis by sample size ( $\mathrm{N}$ subjects $>500$ ), we detected no significant association between the T241M polymorphism and lung cancer risk (TT vs $\mathrm{MM}$ : $\mathrm{OR}=0.74,95 \% \mathrm{CI}=0.49$ 1.14; TT vs MT: OR $=0.90,95 \% \mathrm{CI}=0.78-1.05$; dominant model: $\mathrm{OR}=1.08,95 \% \mathrm{CI}=$ 0.94-1.24; recessive model: $\mathrm{OR}=0.79,95 \% \mathrm{CI}=0.52-1.19)$. The cumulative meta-analyses showed an increasing trend in the estimated risk effect from 2004-2011, which showed that the $\mathrm{T} 241 \mathrm{M}$ polymorphism is not associated with lung cancer risk and that the results were stable (Figure 3).

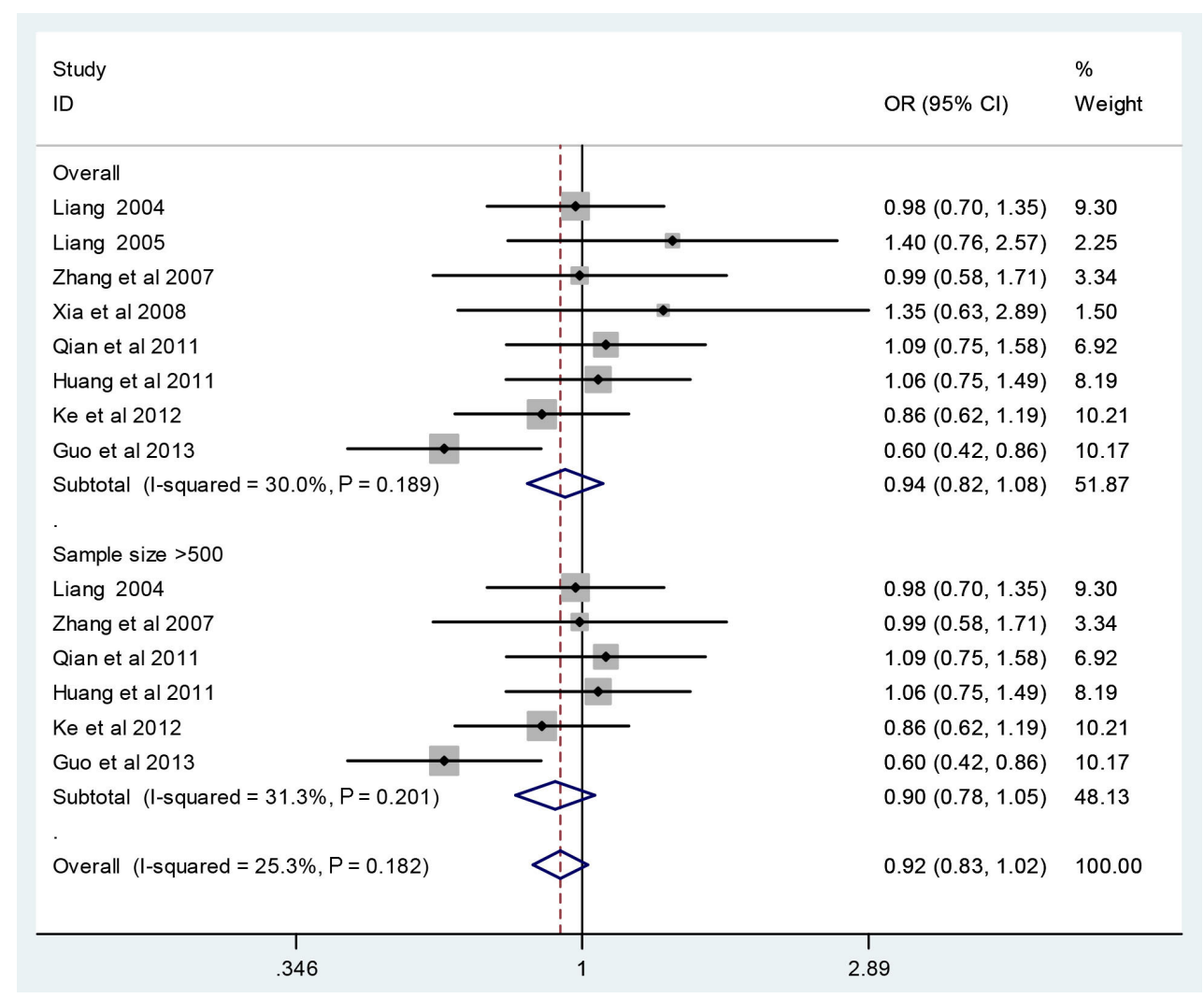

Figure 2. Forest plot of lung cancer risk associated with the XRCC3 T241M polymorphism for TT $v s$ MT. The squares and horizontal lines correspond to the study-specific odds ratios (OR) and 95\% confidence intervals (CI). 


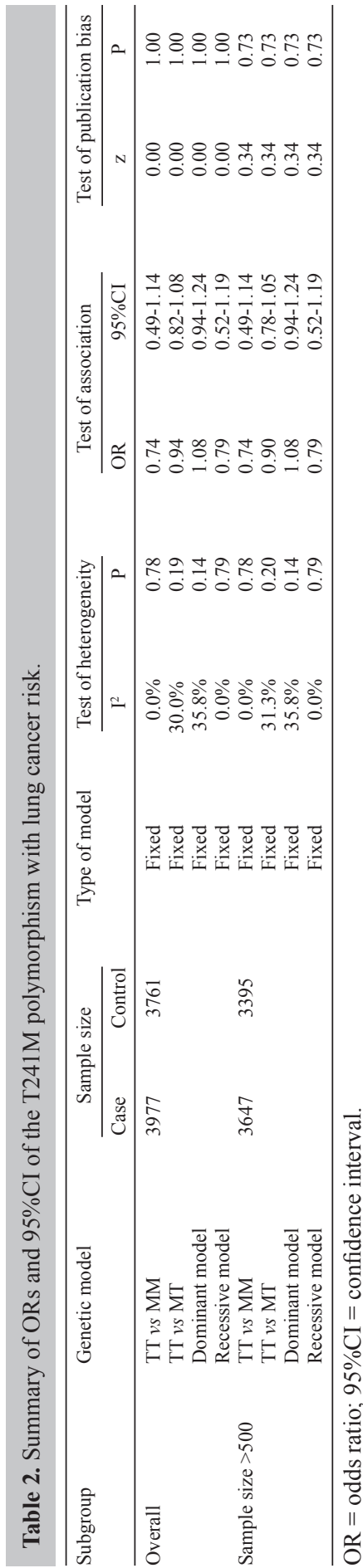




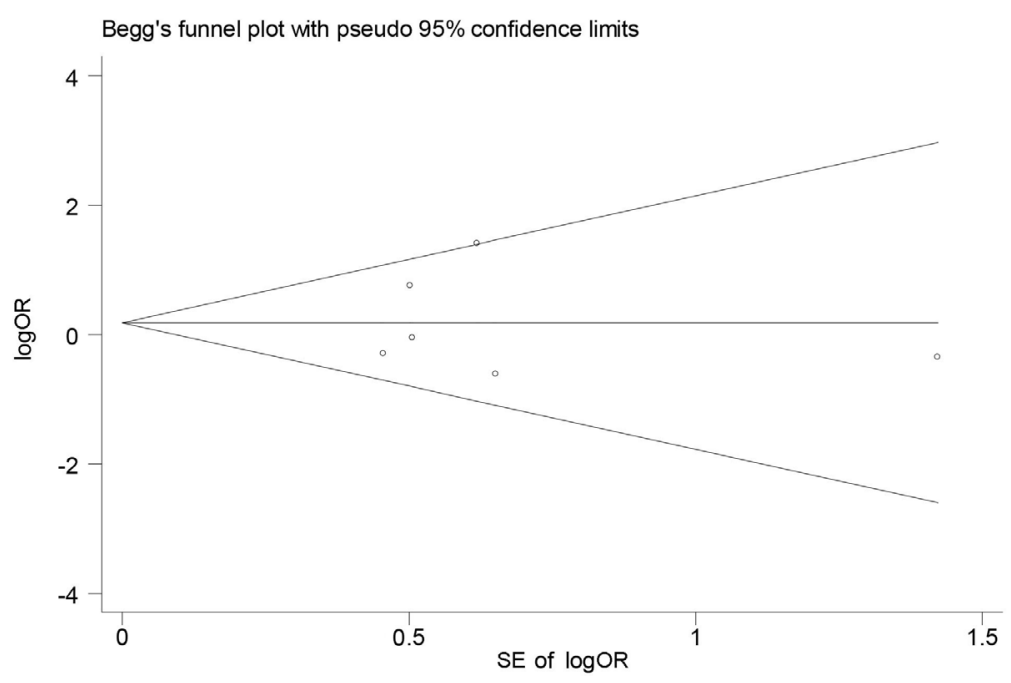

Figure 3. Cumulative meta-analysis for the $X R C C 3 \mathrm{~T} 241 \mathrm{M}$ polymorphism in the fixed-effect pooled odds ratios (OR) with the corresponding confidence interval at $95 \%(95 \% \mathrm{CI})$.

\section{Publication bias and sensitivity analysis}

Begg's funnel plots were generated to assess publication bias in the reports included in the meta-analysis. The shape of funnel plots showed no evidence of publication bias (Figure 4 and Table 2). Sensitivity analysis was conducted to evaluate the influence of each eligible study and changing the regression model on the pooled OR and the overall effect. After omitting individual studies or altering the regression model, the pooled OR and $\mathrm{P}$ value for the overall effect of the null genotype did not significantly change, suggesting that the results of the meta-analysis were stable in these groups.

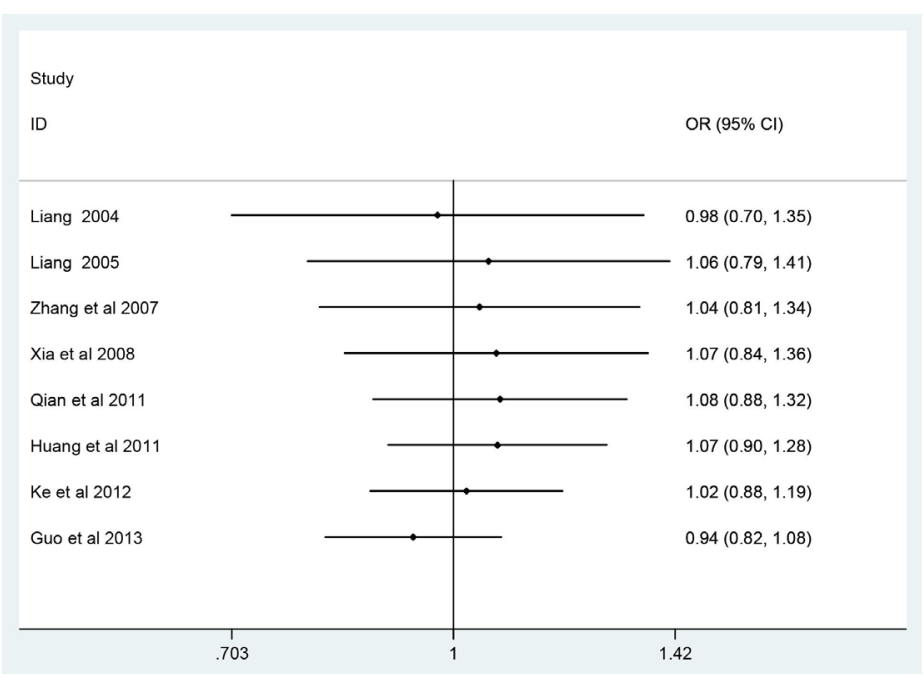

Figure 4. Funnel plot of the XRCC3 T241M polymorphism and susceptibility of lung cancer for TT $v s \mathrm{MT}$. 


\section{DISCUSSION}

It is estimated that China will soon have the world's highest prevalence of lung cancer, with the mortality rate projected to exceed one million by 2025 at the current rate of increase (Ma et al., 2008). Evidence suggests that cancer can be initiated by DNA damage and that most DNA damage can be removed by DNA repair enzymes such as XRCC3. XRCC3 encodes a protein that participates in homologous recombination repair of DSBs. It is a member of an emerging family of Rad-51-related proteins that may take part in homologous recombination to repair DSBs and maintain chromosome stability (Brenneman et al., 2000). Carriers of a variant allele of $X R C C 3, \mathrm{~T} 241 \mathrm{M}$, show relatively high DNA adduct levels in lymphocyte DNA, indicating that this polymorphism is associated with relatively low DNA repair capacity (Matullo et al., 2001a). Previous studies have evaluated the relationship between the T241M polymorphism in the $X R C C 3$ gene and lung cancer risk. However, the results remain controversial. Because individual studies include relatively small numbers of participants and are underpowered for detecting an association, meta-analysis may be an appropriate approach for obtaining a more definitive conclusion. To date, 2 previous meta-analyses were performed to assess the contradictory findings. The results showed that the T241M polymorphism was not associated with the risk of lung cancer (Zhan et al., 2013; Xu et al., 2013). However, studies included in these meta-analyses were primarily conducted in Caucasian populations. In recent years, a larger number of studies have examined the Han Chinese; thus, we performed the present meta-analysis on 8 eligible studies including a total of 3977 lung cancer patients and 3761 controls. The studied population was confined to Chinese individuals with a homogeneous genetic background. In addition, all relevant studies published in English or Chinese were included in the meta-analysis to reduce language biases. The results revealed that the T241M polymorphism is not associated with an increased or decreased risk of lung cancer in Chinese Han populations (TT vs $\mathrm{MM}$ : OR $=0.74,95 \% \mathrm{CI}=0.49-1.14$; TT $v s$ MT: OR $=$ $0.94,95 \% \mathrm{CI}=0.82-1.08$; dominant model: $\mathrm{OR}=1.08,95 \% \mathrm{CI}=0.94-1.24$; recessive model: $\mathrm{OR}=0.79,95 \% \mathrm{CI}=0.52-1.19$ ), which was similar to the findings of previous meta-analyses. Existing evidence regarding cumulative meta-analyses suggested no statistically significant association between the T241M polymorphism and lung cancer risk. In several studies included in our meta-analysis with small samples, there may have been selective bias for finding a relationship between the T241M polymorphism and lung cancer development. Furthermore, we performed subgroup analysis based on a sample size $>500$, which revealed no significant association, suggesting that there was no small study bias in our meta-analysis. However, caution should be exercised when considering this conclusion.

The function of the T241M polymorphism may be affected via gene-gene and geneenvironment interactions. A previous study demonstrated that polymorphisms in both genes (XRCC1 Arg399Gln and XRCC3 T241M) had a synergistic effect, increasing the lung cancer risk (Guo et al., 2013). In addition, tobacco smoke contains procarcinogenic compounds that are metabolized into reactive intermediates and cause DNA damage, which may interact with the T241M polymorphism to result in lung cancer (Wang et al., 2003). Further studies involving larger sample sizes should be conducted to investigate the potential relationships between the effect of environmental factors on the T241M polymorphism and lung cancer risk.

In the meta-analysis, no significant interstudy heterogeneities were observed in the heterogeneity tests, indicating that our results were unbiased; additionally, no obvious publication bias existed in the meta-analysis, as the funnel plots for all comparison models were 
symmetrical. However, there were some limitations to this meta-analysis. First, because of incomplete raw data or publication limitations, some relevant studies could not be included in our analysis. Second, we were unable to address all sources of heterogeneity described in other studies for most polymorphisms, although we could have determined subgroup stratifications analysis for the limited number of published studies. Finally, our systematic review was based on unadjusted data, as the genotype information stratified for the main confounding variables was not available in the original papers and the confounding factors addressed across the different studies were variable.

In conclusion, our meta-analysis revealed no association between the T241M polymorphism and lung cancer risk in Chinese populations. Large-scale case-control and populationbased association studies are warranted to validate the risk identified in the current meta-analysis and investigate the potential gene-gene and gene-environment interactions on lung cancer risk.

\section{REFERENCES}

Ammar M, Bouazizi F, Bouhaha R, Zaraa I, et al. (2012). Association analysis of LCE3C-LCE3B deletion in Tunisian psoriatic population. Arch. Dermatol. Res. 304: 733-738.

Brenneman MA, Weiss AE, Nickoloff JA and Chen DJ (2000). XRCC3 is required for efficient repair of chromosome breaks by homologous recombination. Mutat. Res. 459: 89-97.

Chen W, Zhang S and Zou X (2010a). Estimation and projection of lung cancer incidence and mortality in China. Zhongguo Fei. Ai Za Zhi 13: 488-493.

Chen WQ, Zhang SW, Zou XN and Zhao P (2010b). An analysis of lung cancer mortality in China, 2004-2005. Zhonghua Yu Fang Yi Xue Za Zhi 44: 378-382.

Guo S, Li X, Gao M, Li Y, et al. (2013). The relationship between XRCC1 and XRCC3 gene polymorphisms and lung cancer risk in northeastern Chinese. PLoS One 8: e56213.

Huang M, Chen X, Qiu Y, Fan L, Chen J, et al. (2011). Relationship between XRCC3 gene polymorphisms and lung cancer. Wei Sheng Yan Jiu 40: 187-190.

Jemal A, Bray F, Center MM, Ferlay J, et al. (2011). Global cancer statistics. CA Cancer J. Clin. 61: 69-90.

Ke HG, Li J, Shen Y, You QS, et al. (2012). Prognostic significance of GSTP1, XRCC1 and XRCC3 polymorphisms in non-small cell lung cancer patients. Asian Pac. J. Cancer Prev. 13: 4413-4416.

Liang G (2004). Polymorphisms of DNA Repair Genes and Carcinogen Metabolizing Enzymes Genes and Susceptibility to Lung Cancer. Peking Union Medical College, Beijing.

Liang GY (2005). Studies on Susceptibility Genes of Lung Cancer in Chinese Han Population and Rapid Detection Techniques of Single Nucleotide Polymorphisms. Southeast University, Dhaka.

Ma X, Lin C and Zhen W (2008). Cancer care in China: A general review. Biomed. Imaging Interv. J. 4: e39.

Matullo G, Guarrera S, Carturan S, Peluso M, et al. (2001a). DNA repair gene polymorphisms, bulky DNA adducts in white blood cells and bladder cancer in a case-control study. Int. J. Cancer 92: 562-567.

Matullo G, Palli D, Peluso M, Guarrera S, et al. (2001b). XRCC1, XRCC3, XPD gene polymorphisms, smoking and (32) P-DNA adducts in a sample of healthy subjects. Carcinogenesis 22: 1437-1445.

Qian B, Zhang H, Zhang L, Zhou X, et al. (2011). Association of genetic polymorphisms in DNA repair pathway genes with non-small cell lung cancer risk. Lung Cancer 73: 138-146.

Wang Y, Liang D, Spitz MR, Zhang K, et al. (2003). XRCC3 genetic polymorphism, smoking, and lung carcinoma risk in minority populations. Cancer 98: 1701-1706.

Xia W, Zhang Y, Su D, Shi F (2008). Association of single nucleotide polymorphisms of DNA repair gene XRCC3-241 with non-small cell lung cancer. Zhejiang Med. J. 30: 1291-1293.

Xu YH, Gu LP, Sun YJ, Cheng BJ, et al. (2013). No significant association between the XRCC3 Thr241Met polymorphism and lung cancer risk: a meta-analysis. Tumour Biol. 34: 865-874.

Zhan P, Wang Q, Qian Q and Yu LK (2013). XRCC3 Thr241Met gene polymorphisms and lung cancer risk: a metaanalysis. J. Exp. Clin. Cancer Res. 32: 1.

Zhang ZL, Zhou CC, Zhang J, Tang L, et al. (2007). Relationship between polymorphisms of DNA repair gene XRCC3 and susceptibility to lung cancer. Zhonghua Jie He He Hu Xi Za Zhi 30: 936-940.

Zintzaras E and Lau J (2008). Synthesis of genetic association studies for pertinent gene-disease associations requires appropriate methodological and statistical approaches. J. Clin. Epidemiol. 61: 634-645. 